\title{
Induced pluripotent stem cells derived cardiomyocytes from Duchenne Muscular Dystrophy patients in vitro
}

\author{
Fareeha Faizan Ghori ${ }^{1}$, Mohsin Wahid ${ }^{2}$
}

\begin{abstract}
Objective: This study aimed at the in vitro generation of DMD-cardiomyocytes from patient-specific induced pluripotent stem cells derived from a Pakistani patient for future work on DMD in vitro disease modeling and drug testing for efficacy and toxicity.

Methods: This in vitro experimental study was carried out from December 2018 to January 2019 at Stem Cells and Regenerative Medicine Lab (SCRML) at Dow Research Institute of Biotechnology and Biomedical Sciences (DRIBBS), Dow University of Health Sciences (DUHS) Urine derived DMD-iPSCs were used which had been generated previously from a Pakistani DMD patient who had been selected through nonrandom purposive sampling. These were differentiated towards cardiomyocytes using Cardiomyocytes Differentiation media having specified growth factors and then the molecular characterization of the differentiated cells was done using immunofluorescence.

Results: Pakistani patient's DMD-Cardiomyocytes were generated and their identity was confirmed by positive immunofluorescence for the expression of cardiac markers NKX2-5 and TNNT-2.

Conclusion: This study aimed for in vitro generation of DMD cardiomyocytes for future application in disease modeling, new drug testing for efficacy and toxicity, as well as for drug-testing for tailored personalized therapy. To the best of our knowledge, this was the first time DMD-Cardiomyocytes were generated from Pakistani DMD patients using their own induced pluripotent stem cells.
\end{abstract}

KEYWORDS: Duchenne muscular dystrophy, Human induced pluripotent stem cells, In vitro cardiomyocytes.

How to cite this:

doi: https://doi.org/10.12669/pjms.37.5.3104

Ghori FF, Wahid M. Induced pluripotent stem cells derived cardiomyocytes from Duchenne Muscular Dystrophy patients in vitro. Pak J Med Sci. 2021;37(5):1376-1381. doi: https://doi.org/10.12669/pjms.37.5.3104

This is an Open Access article distributed under the terms of the Creative Commons Attribution License (http://creativecommons.org/licenses/by/3.0), which permits unrestricted use, distribution, and reproduction in any medium, provided the original work is properly cited.

1. Fareeha Faizan Ghori,

2. Mohsin Wahid

Department of Pathology,

Dow International Medical College,

Dow University of Health Sciences, Karachi, Pakistan.

1,2: Dow Research Institute of Biotechnology and Biomedical Sciences,

Dow University of Health Sciences,

Karachi, Pakistan.

Correspondence:

Dr. Mohsin Wahid, MBBS, MPhil (Eng), Ph.D. (Eng),

Dow Research Institute of Biotechnology and Biomedical Sciences,

Dow University of Health Sciences,

Karachi, Pakistan.

Department of Pathology, Dow International Medical College,

Dow University of Health Sciences,

Karachi, Pakistan.

Email: mohsin.wahid@duhs.edu.pk

* Received for Publication:

June 22, 2020

* $1^{\text {st }}$ Revision Received:

* $2^{\text {nd }}$ Revision Received:

* Final Revision Accepted:

July 27,2020

April 8, 2021

April 30, 2021

\section{INTRODUCTION}

The lethal X-linked recessive genetic disorder Duchenne Muscular Dystrophy (DMD) affects one in 3500-5000 live born males; securing its status as the most frequently occurring and severe muscular dystrophy. ${ }^{1}$ It is caused by a lack of the sub-sarcolemmal protein dystrophin in skeletal and cardiac muscles where it serves to normally connect the intracellular contractile machinery to the extracellular matrix. The resulting sarcolemmal fragility in response to mechanical stress, cytoskeletal disruption and mitochondrial dysfunction, culminates in cell death. ${ }^{2}$ Attempts at regeneration are ineffective and fibrosis occurs; resulting in rapidly progressive muscular degeneration and weakness. 
The heart is involved in 25\% DMD individuals at six years of age, $59 \%$ at 10 years, majority by 18 years and eventually in up to $90 \% .^{1,3}$ Death usually occurs at a young age in the late 20s, with $40 \%$ being due to cardiac failure, making it the leading cause. $^{3}$ The cardiac component is magnified as advances in mechanical ventilation techniques mitigate the impact of the respiratory component which is the other main cause. ${ }^{1}$

Initially, decreased systolic function and sinus tachycardia occur. ${ }^{4}$ Although Dilated Cardiomyopathy is thought typical of DMD, Left Ventricular Dysfunction, myocardial fibrosis, arrhythmias (in 44\%), conduction abnormalities in Pukinje fibers, Congestive Heart Failure and cardiac arrest have also been observed. ${ }^{1}$

The dilemma of the DMD patients' situation is that currently, no easily available and universally applicable cure exists. Corticosteroids are customarily used to slow progression and mitigate severity while Angiotensin Converting Enzyme Inhibitors and $\beta$-Blockers provide cardiac support. Novel promising approaches being researched to fill this void include drugs for gene therapy like the stop codon read-through drug Atalauren with accelerated conditional approval in Europe as Translarna, and the exon-skipping drug Eteplirsen with conditional approval in the U.S as Exondys 51.5,6 Other approaches being investigated include geneediting for correction of mutation with CRISPR/ Cas9 or for restoration of the reading frame. ${ }^{7}$

Human cells are better suited for In vitro work than animal cell due to inherent difference in genes and hence morphology and physiology. Thus results from In vitro-studies on human DMD-skeletal muscles have benefited clinical trials in other humans or the same person; but these skeletal muscle therapeutics don't improve the cardiac morbidity due to differences in physiology, rate of RNA production, mRNA levels and turnover, Dystrophin levels, leakiness of membrane, exon-skipping drug half-life and drug-toxicity. ${ }^{3}$ Thus, similar In vitro studies on DMD-cardiomyocytes are being employed to aid clinical trials targeting DMD-cardiomyocytes.

These offer a ray of hope for Pakistani families struggling with multiple children being afflicted by this disabling and lethal disease, as well as for new mutations with no prior family history. Never the less, these new generation therapies are mutation specific; while mutations causing DMD are of various types and $25-33 \%$ are new mutations, so their type and frequency vary geographically. ${ }^{8}$
This means that these therapies, as well as results from DMD studies conducted in other parts of the world, may not be fully relevant or suited to the different mutation profile of the Pakistani patients.

Therefore in vitro patient-specific DMDcardiomyocyte from Pakistani patients are required for disease-modeling, existing drugs' efficacy and toxicity screening, and new drug development; before application of these interventions on Pakistani patients.

Acquiring primary cardiomyocytes through biopsy is an invasive risky procedure and these postnatal adult cardiomyocytes do not expand in vitro. Attempts to aid this have been made by scientists using developmental stage cardiac extracellular matrix to provide growth factors and by overexpression of the cell cycle and proliferation regulator Myc in epicardium; while upstream extra-cardiac endocrine control of epicardial factor involved in cardiac development (Insulin-like growth factor 2) has also been demonstrated. ${ }^{9-11}$ While researchers continue to unravel the cardiac developmental process and understand factors required for efficient in vitro primary cardiomyocytes proliferation, induced pluripotent stem cells (iPSCs) from DMD individuals provide a precious source of in vitroderived DMD cardiomyocytes for the purpose of disease modeling and drug testing. These are more expandable and faced with less ethical issues compared to studies in animal.

Induced pluripotent stem cells are pluripotent cells obtained from cellular reprogramming of somatic cells though forced exogenous expression of pluripotency determining factors. Pluripotent stem cells can potentially give rise to an unlimited number any possible cell type in the adult body, including cardiomyocytes, while simultaneously replenishing their own pool. When somatic cells from a DMD individual are reprogrammed, the generated iPSCs may be expanded greatly while still carrying the DMD mutation present in the original cell and are called DMD-iPSCs. Likewise, so do the cardiomyocytes produced from their differentiation, and are called DMDCardiomyocytes. This study aimed to set the protocol in our laboratory for the in vitro generation of DMD-cardiomyocytes from patientspecific DMD-iPSCs of a Pakistani patient for future research work in Pakistan on DMD disease modeling and drug testing for efficacy and toxicity.

Thus, the first objective of this study was DMDiPSC differentiation towards DMD-Cardiomyocytes 
and the second was molecular characterization of the differentiated cells using Immunostaining to check for cardiac marker expression.

\section{METHODS}

This experimental study involving human cell culture was carried out from December 2018 to January 2019 atStem Cell Research and Regenerative Medicine Lab (SCRML) at Dow Research Institute of Biotechnology and Biomedical Sciences (DRIBBS), Dow University of Health Sciences (DUHS) in Karachi city of Pakistan. Ethical approval was obtained from DUHS's Institutional Review Board (IRB-773/DUHD/Approval/2016/299).

For this study, we obtained DMD-iPSCs generated previously in our laboratory from cellular reprogramming of Urine Derived Cells (UDCs) isolated from the urine of a Pakistani DMD patient. Non-random purposive method had been employed for subject selection for urine collection at DUHS's affiliated clinics; recruiting nine years old male DMD individual, whose diagnosis had been confirmed by neurologists based on his history, clinical findings, muscle biopsy result, serum creatine phosphokinase level, nerve conduction velocity and electromyography findings. Genetic testing had not been done but was part of another study in our laboratory.

Inclusion $\mathcal{E}$ Exclusion Criteria: The inclusion criteria included the male gender and an age of 5-25 years, besides a pre-diagnosed DMD status. The exclusion criteria included known urinary tract tumors, acute-renal failure and dialysis. Informed written consent was obtained from the participant and his guardian.

All work involving cell culture including reagent preparation was performed in a clean room with HEPA-filtered air, inside a class II biosafety cabinet (Thermo Fisher, 1300 Series A2).

First, DMD-iPSCs were plated in a well of a 12 well plate on Geltrex matrix (Gibco, cat\#A1569601) at $10 \mu \mathrm{g} / \mathrm{cm}^{2}$ in $1 \mathrm{ml} /$ well complete Essential 8 medium (Gibco, cat\#A1517001) and incubated in a humidified incubator at $37^{\circ} \mathrm{C}$ and $5 \% \mathrm{CO}_{2}$ (Eppendorf, Galaxy 170R). The iPSCs underwent daily medium change and were expanded to obtain an optimal confluence of $35-60 \%$ for differentiation. The PSC Cardiomyocyte Differentiation Kit (Gibco, cat\#A2921201) consisting of Cardiomyocyte Differentiation Medium A, Cardiomyocyte Differentiation Medium B and Cardiomyocyte Maintenance Medium was used according to the manufacturer's guidelines for the differentiation of iPSCs. Medium was changed very gently on alternate days. On day0 of differentiation, the medium was changed to $1 \mathrm{ml} /$ well Cardiomyocyte Differentiation Medium A, followed by $1 \mathrm{ml} /$ well Cardiomyocyte Differentiation Medium B on day 2 , and $1 \mathrm{ml} /$ well Cardiomyocyte Maintenance Medium on day 4, day 6, day 8 and day 10 . Twice, cells from a single UDC line were reprogrammed and differentiated into cardiomyocytes. Thus, differentiation was repeated twice on $n=1$ patient.

The differentiated cells were checked for the expression of cardiomyocyte markers on day 11 of differentiation using Immunostaining with the Human Cardiomyocyte Immunocytochemistry Kit (Invitrogen, cat\# A25973). The well was fixed for 15 minutes using $0.8 \mathrm{ml} 4 \%$ Formaldehyde in DPBS (Dulbecco's Phosphate Buffered Saline), permeabilized using 1\% Saponin in DPBS and blocked using 3\% Bovine Serum Albumin in DPBS. Incubation with primary antibody diluted to $1 \times$ in blocking solution was done for three hours at room temperature followed by secondary antibody diluted to $1 \times$ in blocking solution for one hour at room temperature. Expression of the cardiomyocyte marker TNNT2 (gene for cardiac Troponin T) was checked using Mouse Anti-TNNT2 Antibody as primary antibody and Donkey Anti-Mouse Alexa Fluor 488 Antibody as secondary antibody. Expression of the cardiomyocyte marker NKX2-5 was checked using Rabbit Anti-NKX2-5 Antibody with Donkey Anti-Rabbit Alexa Fluor 594 Antibody. DAPI (4' 6-Diamidino-2-phenylindole) was used for nuclear staining.

\section{RESULTS}

The morphology of patient specific DMDiPSCs used was observed to comprise of colonies with uniform margins consisting of a relatively homogenous population of spherical cells with large nuclei (high nuclear cytoplasmic ratio) as seen in Fig.1.

Subsequent changes in cellular morphology were observed during the differentiation process. There was a lot of cell death and shedding and the cells appeared opaquer. The left-over differentiated cells lost the typical round, tightly packed iPSC morphology and appeared to be irregularly shaped larger cell bodies with cytoplasmic extension merging into each other with no obvious cell margins in between them (Fig.2).

Molecular characterization of the differentiated cells on day 11 of differentiation demonstrated positive immunofluorescence for the expression of 


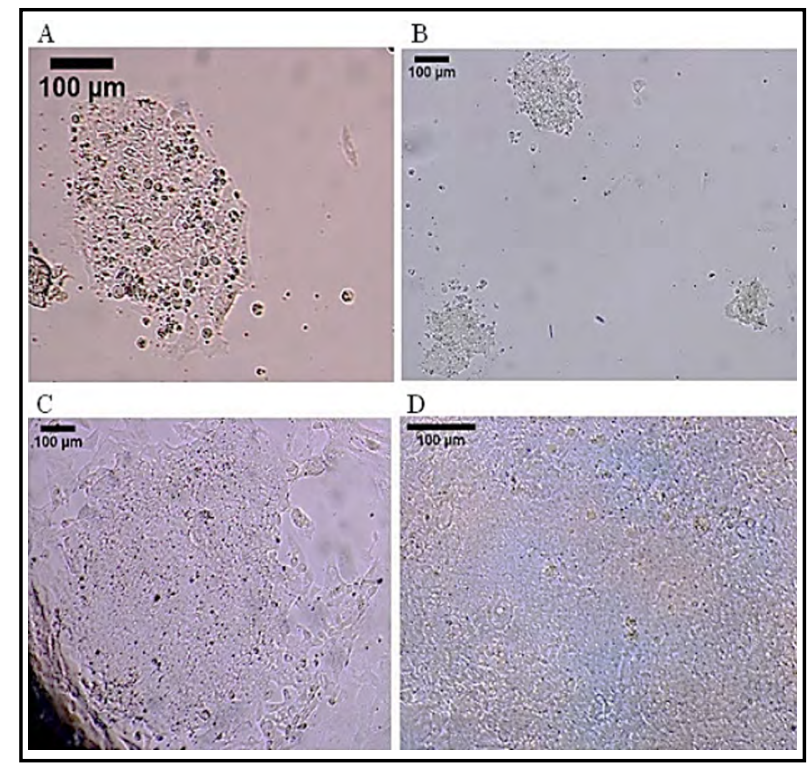

Fig.1: Induced pluripotent stem cell culture:

(A-C) Colonies of iPSCs; (D) Morphology of iPSCs in colony at high power; seen as a homogenous population of spherical cells with large nuclei and high nuclearcytoplasmic ratio. (Scale bar $=100 \mu \mathrm{m})$.

the cardiomyocyte markers TNNT-2 and NKX25. Green immunofluorescence was observed for TNNT-2 while red immunofluorescence was observed for NKX2-5 (Fig.3).

\section{DISCUSSION}

In the past studies, in vitro generation of cardiomyocytes from pluripotent stem cells through spontaneous differentiation in embryoid bodies (aggregates of pluripotent stem cells in suspension), culture with cardio-inductive cells like endoderm-like cell line (END-2) derived from murine embryonal carcinoma, or in media conditioned by them have shown poor efficiency. ${ }^{12}$

Endeavors for unraveling the complex pathways involved in vivo in cardio myogenesis have revealed temporal expression and regulation of transcription factors, mRNAs and growth factors. ${ }^{13}$ Fibroblast Growth Factor (FGF), Bone Morphogenetic Protein (BMP)/ Transforming Growth Factor $\beta$ (TGF $\beta$ ) and Wingless-related mouse mammary tumor virus integration site (Wnt) are the three major pathways recognized as regulators of mesoderm formation and subsequently cardiomyogenesis from it in addition to microRNAs including miR-1 and miR-133. ${ }^{14}$ This knowledge about the in vivo process has been used to designs in vitro protocols for the differentiation of iPSCs towards cardiomyocytes. A popular

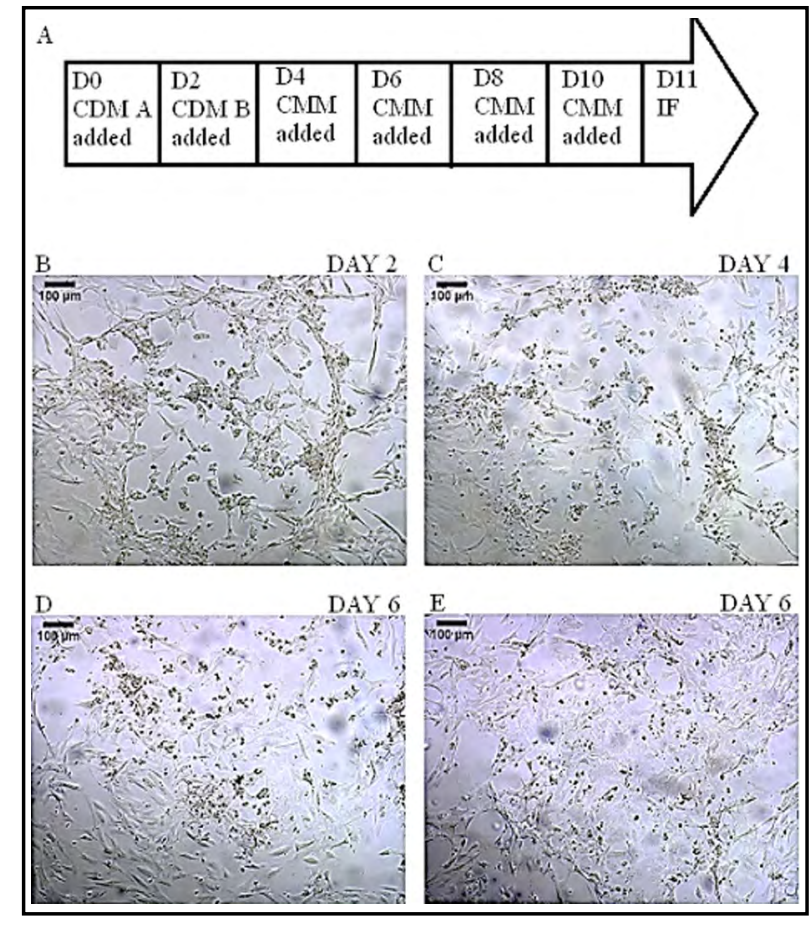

Fig.2: Differentiation of DMD-iPSCs into DMD-cardiomyocytes:

(A) Schematic diagram summarising the workflow; (BE) Changing cellular morphology along with significant cell death and shedding as seen on differentiation Day 2, 4, 6 and 6 respectively. (Scale bar $=100 \mu \mathrm{m}$ ). Key: CDM: Cardiomyocyte Differentiation Medium; CMM: Cardiomyocyte Maintenance Medium; IF: Immunofluorescence.

approach to achieve cardiac differentiation in vitro is the introduction of small molecules in a time and concentration sensitive manner in order to activate and then sequentially inhibit the Wnt pathway as demonstrated by Lian et al. and Sharma at al. ${ }^{15,16}$ Wnt is activated by Glycogen Synthase Kinase$3 \beta$ inhibitors like CHIR99021 that prevent its degradation, and Wnt is inhibited by PORCN inhibitors like IWP2 and Wnt-C59 that prevent its production.

To the best of our knowledge, this is the first time iPSC-derived DMD-Cardiomyocytes have been generated for a Pakistani patient in vitro and no local studies have been published previously on In vitro DMD-cardiomyocytes of Pakistani patients from any source. Very few studies have investigating DMD mutation in Pakistan. Hassan et al. in 2008 found that consistent with geographic variation in DMD mutations, intra-genic deletions were lower in Pakistan (40.75\%), compared to the generally reported level (65\%), which was similar to other Asian nations. ${ }^{17}$ Ansar et al. in 2019 found 


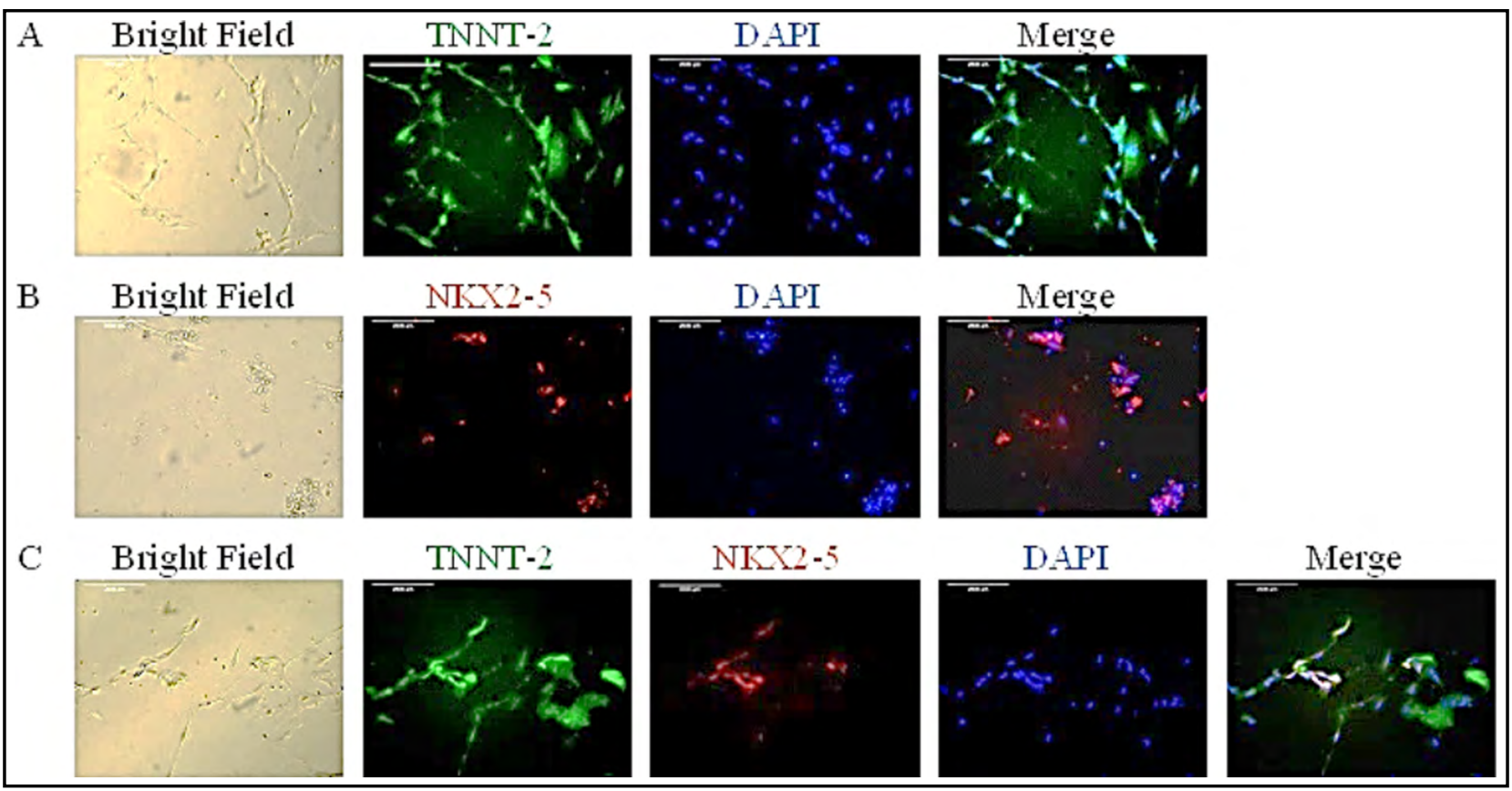

Fig.3: Immunofluorescence of the differentiated cells on day 11 of differentiation for the cardiomyocyte markers TNNT-2 and NKX2-5:

From left to right, bright-field image, immunofluorescence image for the cardiomyocyte marker, blue immunofluorescence image for the nuclear-counter stain DAPI and the merged image for the cardiomyocyte marker with DAPI: (A) Green immunofluorescence for TNNT2 (20×); (B) Red immunofluorescence for NKX2-5 (20×); (C) Green immunofluorescence for TNNT2 and red immunofluorescence for NKX2-5 (20×). (Key: DAPI, 4' 6-Diamidino-2-phenylindole; NKX2-5, NK2 home box 5; TNNT-2, Gene for cardiac Troponin T).

deletions in exon 42-52 (exon 46 most frequent) and duplications in exon 3-7 to be the most frequent in Pakistani patients; while global data reveals exon 45 as the most frequent deletion but exon 2 as the most frequent duplication. ${ }^{18}$

Urine is a suitable non-invasive, painless and convenient source of DMD patients' cells for generating DMD-iPSCs. ${ }^{19}$ Hence, in this study, patient-specific urine-derived DMD-iPSCs have been chosen as starting material and been differentiated towards patient-specific DMDCardiomyocytes, as has been demonstrated previously by others like Guan et al. and Pioner et al. ${ }^{20,21}$ In this study, we have used Gibco's PSC Cardiomyocyte Differentiation Kit. Although knowledge about its constituents is proprietary information, it is intended to simplify the workflow and be more economical than several expensive small molecules purchased individually, as also demonstrated previously by Virtanen et al. and Robert et al. ${ }^{22,23}$

In literature, cardiomyocytes have been reported as expressive of the cardiac markers TNNT-2 and NKX2-5, as well as a-actinin, MLC2a (myosin light chain 2a), MLC2v (myosin light chain 2v) and MYH6 (myosin heavy chain). ${ }^{24,25}$ In this study, identity of the differentiated cells generated has been confirmed as cardiomyocyte by showing expression of the cardiac markers TNNT-2 and NKX2-5.

Limitations of the study: Functional assays for the invitro derived cardiomyocytes including electrophysiological analysis using patch clamp and analysis of mechanical beating behavior were not done due to time and funding limitations.

\section{CONCLUSION}

The non-invasively, painlessly and conveniently sourced urine-derived iPSCs from Pakistani DMD patients can be used to generate patientspecific DMD-Cardiomyocytes using well defined growth media and factors through this simple and economical protocol; for application in drugtesting for tailored personalized therapy, disease modeling, new drug testing for efficacy and toxicity.

Grant Support E Financial Disclosures: None.

Conflict of interest: None. 


\section{REFERENCES}

1. Nguyen $Q$, Yokota T. Antisense oligonucleotides for the treatment of cardiomyopathy in Duchenne muscular dystrophy. Am J Transl Res. 2019;11(3):1202-1218.

2. Esposito G, Carsana A. Metabolic Alterations in Cardiomyocytes of Patients with Duchenne and Becker Muscular Dystrophies. J Clin Med. 2019;8(12):2151. doi: $10.3390 /$ jcm8122151

3. Dick E, Kalra S, Anderson D, George V, Ritso M, Laval $\mathrm{SH}$, et al. Exon skipping and gene transfer restore dystrophin expression in human induced pluripotent stem cells-cardiomyocytes harboring DMD mutations. Stem Cells Dev. 2013;22(20):2714-2724. doi: $10.1089 /$ scd.2013.0135

4. Thomas TO, Morgan TM, Burnette WB, Markham LW. Correlation of heart rate and cardiac dysfunction in Duchenne muscular dystrophy. Pediatr Cardiol. 2012;33(7):1175-1179. doi: 10.1007/s00246-012-0281-0

5. Dworzak J, Frank DE, Charleston JS, Schnell FJ, Voss J, Donoghue C, et al. Quantification of dystrophin to assess the effects of dystrophin-restoring treatments in Duchenne muscular dystrophy: Lessons from the development of eteplirsen. Eur J Paediatr Neurol. 2017;21:e93-e94. doi: 10.1016/j.ejpn.2017.04.1209

6. McDonald CM, Campbell C, Torricelli RE, Finkel RS, Flanigan KM, Goemans N, et al. Ataluren in patients with nonsense mutation Duchenne muscular dystrophy (ACT DMD): a multicentre, randomised, double-blind, placebocontrolled, phase 3 trial. Lancet. 2017;390(10101):1489-1498. doi: 10.1016/S0140-6736(17)31611-2

7. Gee P, Lung MS, Okuzaki Y, Sasakawa N, Iguchi T, Makita $Y$, et al. Extracellular nanovesicles for packaging of CRISPR-Cas9 protein and sgRNA to induce therapeutic exon skipping. Nat Commun. 2020;11(1):1-18. doi: 10.1038/ s41467-020-14957-y

8. Santos R, Goncalves A, Oliveira J, Vieira E, Vieira JP, Evangelista T, et al. New variants, challenges and pitfalls in DMD genotyping: implications in diagnosis, prognosis and therapy. J Hum Genet. 2014;59(8):454-464. doi: 10.1038/ jhg.2014.54

9. Williams C, Quinn KP, Georgakoudi I, Black III LD. Young developmental age cardiac extracellular matrix promotes the expansion of neonatal cardiomyocytes in vitro. Acta Biomater. 2014;10(1):194-204. doi: 10.1016/j. actbio.2013.08.037

10. Shen H, Cavallero S, Estrada KD, Sandovici I, Kumar SR, Makita T, et al. Extracardiac control of embryonic cardiomyocyte proliferation and ventricular wall expansion. Cardiovasc Res. 2015;105(3):271-278. doi: 10.1093/cvr/cvu269

11. Del Campo CV, Lioux G, Carmona R, Sierra R, MunozChapuli R, Claveria $C$, et al. Myc overexpression enhances epicardial contribution to the developing heart and promotes extensive expansion of the cardiomyocyte population. Sci Rep. 2016;6(1):1-12. doi: 10.1038/srep35366

12. Otsuji TG, Minami I, Kurose Y, Yamauchi K, Tada M, Nakatsuji N. Progressive maturation in contracting cardiomyocytes derived from human embryonic stem cells: Qualitative effects on electrophysiological responses to drugs. Stem Cell Res. 2010;4(3):201-213. doi: 10.1016/j. scr.2010.01.002

13. Yassa ME, Mansour IA, Sewelam NI, Hamza H, Gaafar T. The impact of growth factors on human induced pluripotent stem cells differentiation into cardiomyocytes. Life Sci. 2018;196:38-47. doi: 10.1016/j.lfs.2018.01.009
14. Kalra S, Montanaro F, Denning C. Can human pluripotent stem cell-derived cardiomyocytes advance understanding of muscular dystrophies? J Neuromuscul Dis. 2016;3(3):309332. doi: 10.3233/JND-150133

15. Lian X, Zhang J, Azarin SM, Zhu K, Hazeltine LB, Bao X, et al. Directed cardiomyocyte differentiation from human pluripotent stem cells by modulating Wnt/ $\beta$-catenin signaling under fully defined conditions. Nat Protoc. 2013;8(1):162-175. doi: 10.1038/nprot.2012.150

16. Sharma A, Zhang Y, Buikema JW, Serpooshan V, Chirikian $\mathrm{O}$, Kosaric N, et al. Stage-specific effects of bioactive lipids on human iPSC cardiac differentiation and cardiomyocyte proliferation. Sci Rep. 2018;8(1):1-10. doi: 10.1038/s41598018-24954-3

17. Hassan MJ, Mahmood S, Ali G, Bibi N, Waheed I, Rafiq $\mathrm{MA}$, et al. Intragenic deletions in the dystrophin gene in 211 Pakistani Duchenne muscular dystrophy patients. Pediatr Int. 2008;50(2):162-166. doi: 10.1111/j.1442200X.2008.02538.x

18. Ansar Z, Nasir A, Moatter T, Khan S, Kirmani S, Ibrahim $S$, et al. MLPA Analyses Reveal a Spectrum of Dystrophin Gene Deletions/Duplications in Pakistani Patients Suspected of Having Duchenne/Becker Muscular Dystrophy: A Retrospective Study. Genet Test Mol Biomark. 2019;23(7):468-472. doi: 10.1089/gtmb.2018.0262

19. Mulder J, Sharmin S, Chow T, Rodrigues DC, Hildebrandt MR, D'Cruz R, et al. Generation of infant-and pediatricderived urinary induced pluripotent stem cells competent to form kidney organoids. Pediatr Res. 2020;87(4):647-655. doi: 10.1038/s41390-019-0618-y

20. Guan X, Mack DL, Moreno CM, Strande JL, Mathieu J, Shi $\mathrm{Y}$, et al. Dystrophin-deficient cardiomyocytes derived from human urine: new biologic reagents for drug discovery. Stem Cell Res. 2014;12(2):467-480. doi: 10.1016/j.scr.2013.12.004

21. Pioner JM, Guan X, Klaiman JM, Racca AW, Pabon L, Muskheli V, et al. Absence of full-length dystrophin impairs normal maturation and contraction of cardiomyocytes derived from human-induced pluripotent stem cells. Cardiovas Res. 2020;116(2):368-382. doi: $10.1093 /$ cvr/cvz109

22. Virtanen I, Toivanen $M$, Toimela $T$, Heinonen $T$, Tuukkanen S. Direct measurement of contraction force in human cardiac tissue model using piezoelectric cantilever sensor technique. Curr Appl Phys. 2020;20(1):155-160. doi: 10.1016/j.cap.2019.10.020

23. Roberts EG, Kleptsyn VF, Roberts GD, Mossburg KJ, Feng B, Domian IJ, et al. Development of a bio-MEMS device for electrical and mechanical conditioning and characterization of cell sheets for myocardial repair. Biotechnol Bioeng. 2019;116(11):3098-3111. doi: 10.1002/bit.27123

24. Ronaldson-Bouchard K, Ma SP, Yeager K, Chen T, Song L, Sirabella D, et al. Advanced maturation of human cardiac tissue grown from pluripotent stem cells. Nature. 2018;556(7700):239-243. doi: 10.1038/s41586-018-0016-3

25. Zhang JZ, Termglinchan V, Shao N-Y, Itzhaki I, Liu C, Ma $\mathrm{N}$, et al. A Human iPSC Double-Reporter System Enables Purification of Cardiac Lineage Subpopulations with Distinct Function and Drug Response Profiles. Cell Stem Cell. 2019;24(5):802-811.e805. doi: 10.1016/j.stem.2019.02.015

Authors Contribution: FFG conducted all the experiments under supervision and wrote the manuscript. MW designed the study, supervised all the experiments, and performed review and final approval of manuscript. 